\title{
Wave Vector Star Channel Associated with Superstructure of Crystal and Bravais Lattice Types
}

\author{
Il-Hwan Kim ${ }^{1}$, Jong-Ok Pak ${ }^{2}$, Un-Son Jong ${ }^{3}$, Song-on Kim ${ }^{4}$, Chol-Gyong Kim ${ }^{5}$, Song-Il Ri ${ }^{6}$ \\ 1,3,4 Department of physics, Kim Hyong Jik Normal University, Pyongyang Democratic People's Republic of Korea \\ ${ }^{2}$ Physics chair, Pyongyang University of Mechanical engineering, Pyongyang Democratic People's Republic of Korea \\ ${ }^{5,6}$ Department of Mathematics, University of Science, Pyongyang, D. P. R. of Korea
}

\begin{abstract}
For developing new materials, high superconductor, ferroics such as ferroelectric, ferromagnetic, ferroelastic, and multi-ferroics such as ferroelectric- magnetic, mageneto-elastic, and so on, and for studying their features, the study of the symmetry is especially important. As well-known, all crystals belong to any group among 230 space groups. In these crystals, change of physical properties is related with the breaking of rotation, translational symmetry and is described to full irreducible representation of space group. For a long time, many researchers have been studying the possible superstructure that is practically significant. The superstructure is obtained during the translational symmetry breaking. The study of the superstructure phases aims at finding out the lattice types of the possible lower symmetry phases during translational symmetry breaking. This is directly associated with study of the wave vector star in reciprocal lattice space.
\end{abstract}

Keywords: Wave vector star channel, star channel group, translational symmetry breaking, Bravais lattice

\section{Introduction}

For developing new materials, high superconductor, ferroics such as ferroelectric, ferromagnetic, ferroelastic, and multi-ferroics such as ferroelectric- magnetic, mageneto-elastic, and so on, and for studying their features, the study of the symmetry is especially important.

As well-known, all crystals belong to any group among 230 space groups. In these crystals, change of physical properties is related with the breaking of rotation, translational symmetry and is described to full irreducible representation of space group.

For a long time, many researchers have been studying the possible superstructure that is practically significant. The superstructure is obtained during the translational symmetry breaking. The study of the superstructure phases aims at finding out the lattice types of the possible lower symmetry phases during translational symmetry breaking. This is directly associated with study of the wave vector star in reciprocal lattice space.

The pioneer in this was Lifshitz ${ }^{[1]}$. In 1941, in connection with the translational symmetry breaking, Lifshitz studied systematically the possible Bravais lattice types during the second-order phase transition in crystal. Bravais lattices are obtained using the full irreducible representation of space group.

After that, a variety phases transitions, that is, not only the second-order phase transition but also "the first-order phase transition near the second-order" were found out in various crystals and study of phase transition had been deepened. As a result, it was necessary to fill in and complete the result which Lifshitz had obtained.

So, in 1976, to use more effectively the result of Lifshitz, $\mathrm{Naish}^{[2]}$ accepted the conception of the wave vector star channel and using the conception identified concretely Bravais lattice $^{[2,3]}$.

From this time, the conception of the star channel has been used widely for studying the phase transition. For example, it was used for analyzing the superstructure of an alloy and magnetic structure of a magnetic substance ${ }^{[4 \sim 10]}$. Also star channel was used for analyzing the structure of superstructure phase during the ordering of vacancies in nonstoichiometric compounds.

In paper ${ }^{[3]}$, we can show only lattice type of high symmetry which is obtained during phase transition.

Table 1 shows the wave vector star channels and lattice types of cubic crystals among the result of paper ${ }^{[1]}$ and ${ }^{[3]}$.

Table 1: Wave vector star channels and lattice types of cubic

\begin{tabular}{|c|c|c|c|c|c|}
\hline \multicolumn{6}{|c|}{ Result of paper [3] } \\
\hline \multicolumn{4}{|c|}{ Result of paper [1] } & & \\
\hline$G$ & $G_{\alpha}$ & Vector of Bravais unit & $\mathrm{n}$ & $\boldsymbol{k}$ & Arm of Star \\
\hline \multirow{6}{*}{$\Gamma_{c}^{f}$} & $\Gamma_{q}$ & $\square\left(a_{1}+a_{2}\right), \square\left(a_{1}-a_{2}\right), a_{3}$ & 2 & $\boldsymbol{k}_{10}(3)$ & (i) \\
\hline & $\Gamma_{r h}$ & $a_{1}+\square\left(a_{2}+a_{3}\right), a_{2}+\square\left(a_{1}+a_{3}\right), a_{3}+\square\left(a_{1}+a_{2}\right)$ & 2 & $k_{9}(4)$ & (i) \\
\hline & $\Gamma_{c}$ & $a_{1}, a_{2}, a_{3}$ & 4 & $\boldsymbol{k}_{10}(3)$ & $(i j),(123)$ \\
\hline & {$\left[\Gamma_{m}\right]$} & $a_{1}+a_{2,} a_{1}-a_{2}, \square\left(a_{2}+a_{3}\right)$ & & & \\
\hline & {$\left[\Gamma_{o}^{b}\right]$} & $2 a_{1}, a_{2}+a_{3}, \square\left(a_{2}-a_{3}\right) ; a_{1}+\square\left(a_{2}+a_{3}\right)$ & 4 & $\boldsymbol{k}_{9}(4)$ & $(i j)$ \\
\hline & $\Gamma_{q}^{v}$ & $a_{1}, a_{2}, 2 a_{3} ; a_{3}+\square\left(a_{1}+a_{2}\right)$ & 4 & $\boldsymbol{k}_{8}(6)$ & $(i),(12)(34)(56)$ \\
\hline
\end{tabular}

Volume 5 Issue 7, July 2016 www.ijsr.net

Licensed Under Creative Commons Attribution CC BY 
International Journal of Science and Research (IJSR)

ISSN (Online): 2319-7064

Index Copernicus Value (2013): 6.14 | Impact Factor (2015): 6.391

\begin{tabular}{|c|c|c|c|c|}
\hline$\Gamma_{c}^{f}$ & $2 a_{1}, 2 a_{2}, 2 a_{3} ; a_{2}+a_{3}, a_{1}+a_{2}, a_{1}+a_{3}$ & 8 & $\boldsymbol{k}_{9}(4)$ & $(i j k),(1234)$ \\
\hline$\Gamma_{q}$ & $2 a_{1}, 2 a_{2}, a_{3}$ & 16 & $\boldsymbol{k}_{8}(6)$ & $\begin{array}{c}(1234),(1256),(3456) \\
(123)(124)(125)(126) \\
(134)(156)(234)(256) \\
(346)(356)(456)(13)(14)(15)(16)(23)(24)(25)(26) \\
(35)(36)(45)(46)\end{array}$ \\
\hline$\Gamma_{c}$ & $2 a_{1}, 2 a_{2}, 2 a_{3}$ & 32 & $\boldsymbol{k}_{8}(6)$ & $\begin{array}{c}\text { (135) (136) }(145)(146) \\
(235)(236)(245)(246) \\
(i j k l) ; \text { except }(1234),(1256),(3456) \\
(\text { ijklm }),(123456)\end{array}$ \\
\hline
\end{tabular}

In table, first and second columns describe each other lattice types of the high and lower symmetry phase and fourth and fifth columns describe each other the change of volume and the number of star. In sixth column number in brackets expresses the number of arms of given star. In second column, lattice type in a sign [ ] shows that results of the paper [3] differ from the paper [1].

But it was known that not only lattices of the high symmetry but also lattices of the lower symmetry were possible during the phase transition.

So, in 1983, jaric ${ }^{[11]}$ accepted the conception of the wave vector substar, he called substar the combination of all the possible arms of wave vector star. Using this conception he suggested a method finding out the space group of the lower symmetry. He also defined the little group of substar that it is set of elements of space group leaving a substar invariant.

In paper [11], he showed that one could find out the space group of the lower symmetry by subduing into the subgroup of the little group of the substar in the full irreducible representation of the space group.

By using previous results, we can know not only the possible lattice types but also distinct arms of the wave vector star during the spontaneous symmetry breaking.

But, when we apply the star channel to the study of the phase transition, we can know that the conception of star channel defined in the previous paper is in sufficient. In paper [2,3] and paper [11], the conception star channel and substar is essentially equal. So the wave vector star channel shown in paper [2, 3] have some "unnecessary" channels not to be tolerated by the translational symmetry. For example, there are some star channels in face-centered cubic lattice. In case of Lifshitz star $\boldsymbol{k}_{8}$, one arm channel ( $i$ )of the star, channels of two $\operatorname{arms}(13),(14),(15),(16),(23),(24),(25)$, (26), (35), (36), (45), (46), channels of three arms (123), (124), (125), (126), (134), (156), (234), (256), (346), (356), (456), (135), (136), (145), (146), (235), (236), (245), (246),(135), channels of four arms $(i j k l)$ except (1234), (1256), (3456), channels of five arms (ijklm) and so on, unnecessary channels which have nothing to do with spontaneous symmetry breaking.

Preceding researchers didn't consider wave vector star channel in relation with symmetry of order parameter. They regarded the star channel as the combination of all the arms of star satisfying the condition of the translational symmetry $e^{-i \boldsymbol{k}(\alpha) \boldsymbol{t}_{D}}=1$
As shown in table -1, the star channels consisting of two, three, four, five, six arms of Lifshitz star $\boldsymbol{k}_{8}$ are related to primitive cubic whose volume change is 32 times as many as cubic lattice. $2 a_{1}, 2 a_{2}, 2 a_{3}$ are the translation vectors of Bravais cell of the lower symmetry phase. This vectors satisfy the condition of the translational symmetry when above star channel is given.

But, generally each star channelsreveal the different properties of the translational symmetry breaking. Thus results mentioned above did not correct.This is directly associated with limitation of method for determining the star channel. Therefore, in this paper, we are going to establish the method determining the wave vector star channel.

On the other hands, in paper [3], researcher defined star channel as a set of arms of star taking part in phase transition or as Bravais lattice types determined by them, and they call them "transition channel" or "phase transition channel" After all, they didn't consider wave vector star channel in relation with symmetry of order parameter. During the translational symmetry breaking, there are many possible phases of not only high symmetry but also lower symmetry. These all phases are induced from a wave vector star channel. Thus, it does not exact that the wave vector star is "phase transition channel".

So, in this paper, we considered the wave vector star channel associated with the translational symmetry breaking of crystal in close relation with of order parameter, different from the preceding studies and investigated a kind of the new method for obtaining it. Also, the possible Bravais lattices are investigated.

\section{Method for determining the wave vector star channel}

Conveniently, we can write as follows the change $\delta \rho$ in the distribution density function of the new phase when phase transitions take place according to certain irreducible representation of crystal symmetry group $G$.

$$
\delta \rho(\boldsymbol{r})=\sum_{\alpha=1}^{m} \sum_{\beta=1}^{l} C_{j \beta}^{(\alpha)} \varphi_{j \beta}^{(\alpha)}(\boldsymbol{r})
$$

where $\alpha$ is the number of arms of the wave vector star, $\beta$ is the number of basis functions of the $\mathrm{jth}, \mathrm{j}$-dimensional irreducible representation of the wave vector group $G_{k(\alpha)}$ which is related to an arm $\boldsymbol{k}(\alpha)$ of star $\boldsymbol{k}$

Since the transition occurs along particular arms of star, we can write the following equation ${ }^{[12]}$.

$$
\delta \rho(\boldsymbol{r})=\sum_{\alpha=1}^{m} C^{(\alpha)} \varphi^{(\alpha)}(\boldsymbol{r})=\sum_{\alpha=1}^{m} \delta \rho_{\alpha}(\boldsymbol{r})
$$




\section{International Journal of Science and Research (IJSR) \\ ISSN (Online): 2319-7064}

Index Copernicus Value (2013): 6.14 | Impact Factor (2015): 6.391

From the condition of the translational symmetry ,the following equation is obtained.

$$
e^{-i \boldsymbol{k}(\alpha) \boldsymbol{t}_{D}}=1, \quad(\alpha=1 \sim m)
$$

After all,crystal lattice of the new phase is characterized by translation vector $\boldsymbol{t}_{D}$ satisfying the equation (3) with respect to the given wave vector star. But this equation is only satisfied with respect to the non-zero arms of star corresponding to the non-zero order parameter. Therefore, equation (3) is not the enough condition but is necessary condition for determining the wave vector star channel. After all, the problem determining the star channel is finding out the order parameter.

So, in this paper, we accepted the new space associated with the arms of star by corresponding one-to-one the arm of wave vector star to the order parameter. Then this is space of the order parameter spanned by the subdued representation into the translation group in the full irreducible representation.

In the equation $(2), \varphi^{(\alpha)}(\boldsymbol{r})$ is the basis function of representation of the translation group $T$. After all, $C^{(\alpha)}$

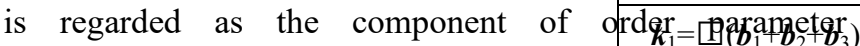
characterizing the translational symmetry bueaking. Beeause the different arms of star can take part in the stitletthal phase transition, $\left\{C^{(\alpha)}\right\}$ becomes multi-complenent $\left(b_{\text {or }}\right.$ ber parameter and is changedaccording to the redpreseftathon $\Gamma(\boldsymbol{t})$ of translation group $T$.

monoclinicP $\left(\Gamma_{m}\right)$

Now introduce the representation space $\varepsilon_{m}$ spanned by $\Gamma(\boldsymbol{t})$ and establish the orthogonal coordinate system with $C^{(\alpha)}$ component in this space. We call this m-dimengional

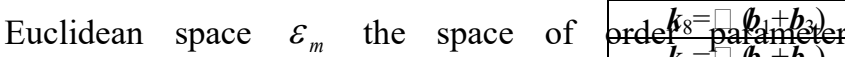

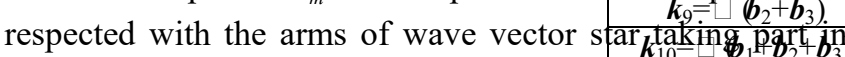
the translational symmetry breaking. Then, in this space, $C^{(\alpha)}$ is the projection into coordinate axis $e_{1}, e_{2}, \cdots, e_{m}$ of the multidimensional vector $C$.

star

Meanwhile, infinitude group $T$ and it s representation spanned by $\varepsilon_{m}$-space could be considered to finithude group $T_{f}=T / \operatorname{ker} \Gamma$ and faithful representation $\Gamma \boldsymbol{k}_{8}=\square$. $\Gamma_{f}$ is Unitary representation, the set of the $=$ mallnix $\boldsymbol{b}_{\mathrm{es}}+\boldsymbol{b}_{\mathrm{o}} \mathrm{f}$ $\Gamma_{f}$ in the $\varepsilon_{m}$-space is a certain multidimensional abstract point group, we call this group translation $L$-group. Then the wave vector star channel can be found out with a method of finding non-zero invariant subspacestaf the order parameter space which is invariant to all the subgroups effannel the translation $L$-group.

The dimension of this space can be found ont the $\boldsymbol{b}_{0}$ ollowing $\boldsymbol{k}_{2}$ equation. part in the phase transition. groups

Triclinic $(\Gamma \mathrm{t})$
In above expression, $[H]$ is the order of subgroup of the translation group, $\chi\left(h_{i}\right)$ is the sum of character of the representation respected with all the elements of subgroup, $r$ is the number of the arms of the wave vector star channel. This dimension just corresponds to the number of arms of wave vector star taking part in the phase transition.

Thus, similarly to paper [13], we can define the set of invariant vector of the translation $L$-group and can find out the star channels corresponding to it. The arms contained in this channel become the just arms of star taking

In this paper, we obtained 179 wave vector star channels associated with 80 Lifshitz stars of 230 space groups. We showed the result in table-2, in contrast to paper [3].

Table 2: Channelsof Lifshitz Wave vector stars of 230 space

\begin{tabular}{|c|c|c|c|c|c|}
\hline \multicolumn{2}{|c|}{ Result of [3] } & Result of this & \multirow{2}{*}{$\begin{array}{c}\text { star } \\
\text { channel }\end{array}$} & \multicolumn{2}{|c|}{ Result of [3] } \\
\hline channel & $\mathrm{n}$ & {$[k]$} & & $\mathrm{n}$ & \\
\hline $\boldsymbol{k}_{1}$ & 1 & \multirow{4}{*}[k]{} & $\boldsymbol{k}_{5}=\square \boldsymbol{b}_{1}$ & $\boldsymbol{k}_{5}$ & \multirow{4}{*}{2} \\
\hline $\boldsymbol{k}_{2}$ & \multirow{3}{*}{2} & & $\boldsymbol{k}_{6}=\square \boldsymbol{b}_{2}$ & $\boldsymbol{k}_{6}$ & \\
\hline $\boldsymbol{k}_{3}$ & & & $\boldsymbol{k}_{7}=\square \boldsymbol{b}_{3}$ & $\boldsymbol{k}_{7}$ & \\
\hline $\boldsymbol{k}_{4}$ & & & $\boldsymbol{k}_{8}=0$ & $\boldsymbol{k}_{8}$ & \\
\hline
\end{tabular}

\begin{tabular}{|c|c|c|c|c|c|c|}
\hline \multicolumn{2}{|c|}{ Result of [3] } & Result of this & \multirow{2}{*}{$\begin{array}{c}\text { star } \\
\text { channel }\end{array}$} & \multicolumn{2}{|c|}{ Result of [3] } & \\
\hline channel & $\mathrm{n}$ & {$[k]$} & & $\mathrm{n}$ & & \\
\hline $\boldsymbol{k}_{7}$ & 1 & \multirow{4}{*}[k]{} & $\boldsymbol{k}_{11}=\square \boldsymbol{b}_{3}$ & $\boldsymbol{k}_{11}$ & \multirow{4}{*}{2} & \\
\hline $\boldsymbol{k}_{8}$ & \multirow{3}{*}{2} & & $\boldsymbol{k}_{12}=\square \boldsymbol{b}_{1}$ & $\boldsymbol{k}_{12}$ & & \\
\hline $\boldsymbol{k}_{9}$ & & & $\boldsymbol{k}_{13}=\square \boldsymbol{b}_{2}$ & $\boldsymbol{k}_{13}$ & & \\
\hline $\boldsymbol{k}_{10}$ & & & $\boldsymbol{k}_{14}=\square\left(\boldsymbol{b}_{1}+\boldsymbol{b}_{2}\right)$ & $\boldsymbol{k}_{14}$ & & \\
\hline
\end{tabular}

monoclinic $\mathrm{A}\left(\Gamma_{m}{ }^{b}\right)$

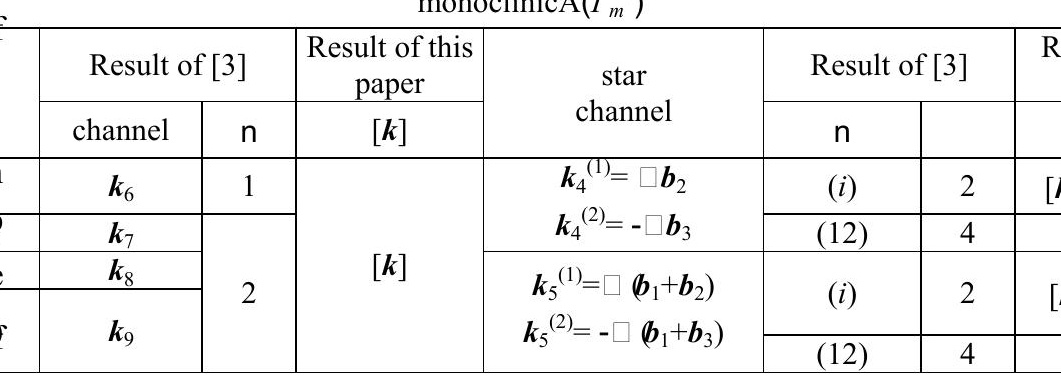

Orthorhombic $\mathrm{P}\left(\Gamma_{o}\right)$

\begin{tabular}{|c|c|c|c|c|c|c|}
\hline \multicolumn{2}{|c|}{${ }_{r}^{a}$ Result of [3] } & $\begin{array}{c}\text { Result of this } \\
\text { paper }\end{array}$ & \multirow{2}{*}{$\begin{array}{c}\text { star } \\
\text { channel }\end{array}$} & \multicolumn{2}{|c|}{ Result of [3] } & $\mathrm{Re}$ \\
\hline fannel & $\mathrm{n}$ & {$[k]$} & & $\mathrm{n}$ & & \\
\hline $\boldsymbol{k}_{19}$ & 1 & \multirow{4}{*}[k]{} & $\boldsymbol{k}_{23}=\square\left(\boldsymbol{b}_{2}+\boldsymbol{b}_{3}\right)$ & $\boldsymbol{k}_{23}$ & \multirow{4}{*}{2} & \\
\hline $\boldsymbol{k}_{20}$ & \multirow{3}{*}{2} & & $\boldsymbol{k}_{24}=\square\left(\boldsymbol{b}_{1}+\boldsymbol{b}_{3}\right)$ & $\boldsymbol{k}_{24}$ & & \\
\hline $\boldsymbol{k}_{21}$ & & & $\boldsymbol{k}_{25}=\square\left(\boldsymbol{b}_{1}+\boldsymbol{b}_{2}\right)$ & $\boldsymbol{k}_{25}$ & & \\
\hline $\boldsymbol{k}_{22}$ & & & $\boldsymbol{k}_{26}=\square\left(\boldsymbol{b}_{1}+\boldsymbol{b}_{2}+\boldsymbol{b}_{3}\right)$ & $\boldsymbol{k}_{26}$ & & \\
\hline
\end{tabular}

$$
r=\frac{1}{[H]} \sum_{i} \chi\left(h_{i}\right)
$$

Orthorhombic C $\left(\Gamma_{o}^{b}\right)$

\begin{tabular}{l|l|r} 
Result of [3] & Result of this & star \\
\hline
\end{tabular}




\section{International Journal of Science and Research (IJSR) \\ ISSN (Online): 2319-7064}

Index Copernicus Value (2013): 6.14 | Impact Factor (2015): 6.391

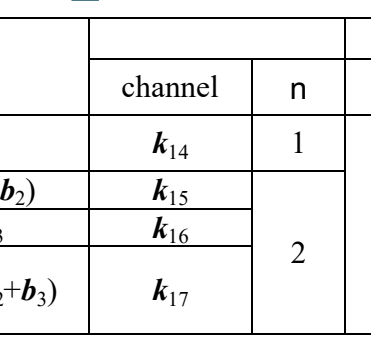

paper

\begin{tabular}{c} 
channel \\
$\boldsymbol{k}_{12}{ }^{(1)}=\square \boldsymbol{b}_{1}$ \\
$\boldsymbol{k}_{12}{ }^{(2)}=-\square \boldsymbol{b}_{2}$ \\
\hline $\boldsymbol{k}_{13}{ }^{(1)}=\square\left(\boldsymbol{b}_{1}+\boldsymbol{b}_{3}\right)$ \\
$\boldsymbol{k}_{13}{ }^{(2)}=-\square\left(\boldsymbol{b}_{2}+\boldsymbol{b}_{3}\right)$
\end{tabular}

\begin{tabular}{|c|c|}
\hline \multicolumn{2}{|c|}{} \\
\hline $\mathrm{n}$ & \\
\hline$(i)$ & 2 \\
\hline$(12)$ & 4 \\
\hline$(i)$ & 2 \\
\hline$(12)$ & 4 \\
\hline
\end{tabular}

\begin{tabular}{|c|c|c|}
\hline \multicolumn{2}{|c|}{ Result of [3] } & Result of this \\
\hline hannel & $n$ & {$[k]$} \\
\hline $\boldsymbol{k}_{14}$ & 1 & \multirow{4}{*}[k]{} \\
\hline $\boldsymbol{k}_{15}$ & \multirow{3}{*}{2} & \\
\hline $\boldsymbol{k}_{16}$ & & \\
\hline $\boldsymbol{k}_{17}$ & & \\
\hline
\end{tabular}

Orthorhombic $\mathrm{F}\left(\Gamma_{o}^{f}\right)$

\begin{tabular}{|c|c|c|c|}
\hline & \multicolumn{3}{|c|}{ Trigonal $\mathrm{R}\left(\Gamma_{r h}\right)$} \\
\hline channel & \multicolumn{2}{|c|}{ Result of [3] } & Result of this paper \\
\hline$\left[\boldsymbol{k}_{12}{ }^{(1)}\right],\left[\boldsymbol{k}_{12}{ }^{(2)}\right]$ & channel & $\mathrm{n}$ & {$[k]$} \\
\hline 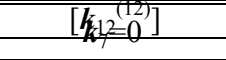 & $\boldsymbol{k}_{7}$ & 1 & \multirow{2}{*}[k]{} \\
\hline $\boldsymbol{k}_{\mathrm{d}}\left[\boldsymbol{k}_{13}\left(\boldsymbol{b}_{1,}, \boldsymbol{b}_{\mathrm{b}_{3}}+\boldsymbol{b}_{3}\right)\right.$ & $\boldsymbol{k}_{8}$ & 2 & \\
\hline $\boldsymbol{k}_{5}^{(1)}\left[\boldsymbol{k}_{13}^{(12)}\left(\boldsymbol{b}_{1}+\boldsymbol{h}_{2}\right)\right.$ & (i) & 2 & {$\left[\boldsymbol{k}_{5}{ }^{(1)}\right],\left[\boldsymbol{k}_{5}{ }^{(2)}\right],\left[\boldsymbol{k}_{5}{ }^{(3)}\right]$} \\
\hline $\boldsymbol{k}_{5}^{(2)}=\square\left(\boldsymbol{b}_{1}+\boldsymbol{b}_{3}\right)$ & $(i j)$ & \multirow{2}{*}{4} & $(*)$ \\
\hline $\boldsymbol{\kappa}_{5}$ & $(123)$ & & {$\left[\boldsymbol{k}_{5}^{(123)}\right]$} \\
\hline
\end{tabular}

star channel

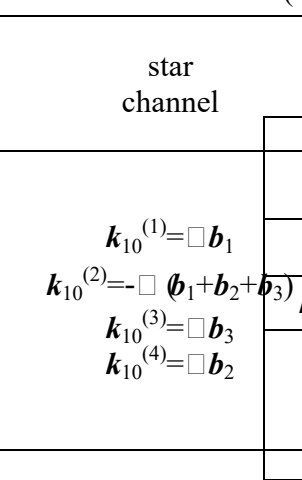

Orthorhombic $I\left(\Gamma \boldsymbol{k}_{1}^{v}\right)^{(1)}=\square\left(\boldsymbol{b}_{1}+\boldsymbol{b}_{2}\right)$

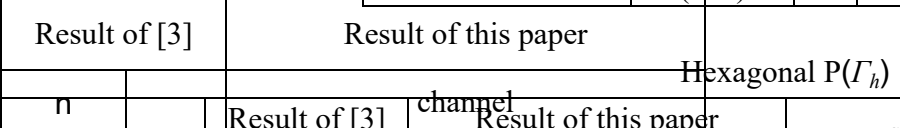

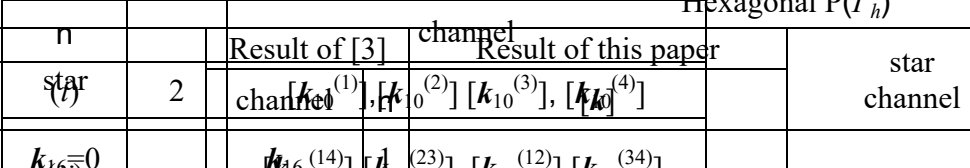

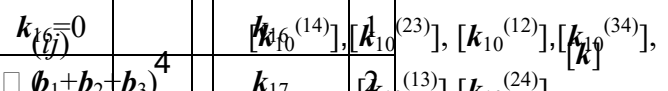

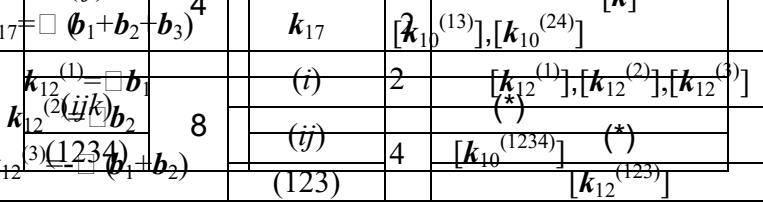

\begin{tabular}{|c|c|c|}
\hline$\left(\Gamma \boldsymbol{k}_{13}^{v}{ }^{(1)}=\square\left(\boldsymbol{b}_{1}+\boldsymbol{b}_{2}\right)\right.$ & (i) & $\left({ }^{*}\right)$ \\
\hline $\begin{array}{l}\boldsymbol{k}_{13}{ }^{(2)}=-\boldsymbol{k}_{13}{ }^{(1)} \\
\text { star }\end{array}$ & Resul(t12f) [3] & $\left.\begin{array}{c}\text { Result of this } \\
\text { paper }\end{array} \boldsymbol{k}_{13}{ }^{(1)}\right]$ \\
\hline & $\mathrm{n}$ & channel \\
\hline
\end{tabular}

$\left(^{*}\right)$

\begin{tabular}{|l|c|c|c|}
\hline \multirow{2}{*}{} & \multicolumn{2}{|c|}{ Result of [3] } & $\begin{array}{c}\text { Result of this } \\
\text { paper }\end{array}$ \\
\cline { 2 - 4 } & channel & $\mathrm{n}$ & {$[\boldsymbol{k}]$} \\
\hline 0 & $\boldsymbol{k}_{17}$ & 1 & \multirow{2}{*}[\boldsymbol{k}]{} \\
\hline $\left.\boldsymbol{b}_{2}+\boldsymbol{b}_{3}\right)$ & $\boldsymbol{k}_{18}$ & 2 & \\
\hline $\boldsymbol{b}_{1}$ & $(i)$ & 2 & {$\left[\boldsymbol{k}_{13}{ }^{(1)}\right],\left[\boldsymbol{k}_{13}{ }^{(2)}\right]$} \\
\cline { 2 - 4 } $\left.\boldsymbol{b}_{2}-\boldsymbol{b}_{3}\right)$ & $(12)$ & 4 & {$\left[\boldsymbol{k}_{13}{ }^{(12)}\right]$} \\
\hline $\boldsymbol{b}_{2}$ & $(i)$ & 2 & {$\left[\boldsymbol{k}_{14}{ }^{(1)}\right],\left[\boldsymbol{k}_{14}{ }^{(2)}\right]$} \\
\hline $\left.\boldsymbol{b}_{1}-\boldsymbol{b}_{3}\right)$ & $(12)$ & 4 & {$\left[\boldsymbol{k}_{14}{ }^{\left({ }^{(12}\right]}\right]$} \\
\hline
\end{tabular}

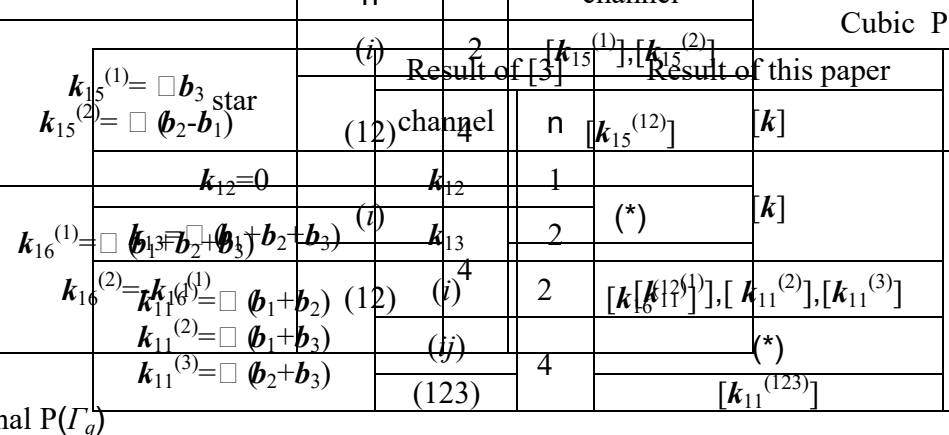

\section{$\boldsymbol{k}_{14}^{(1)}=\square\left(\boldsymbol{b}_{1}+\boldsymbol{b}_{3}\right)$}

$\boldsymbol{k}_{14}{ }^{(2)}=\square\left(\boldsymbol{b}_{2}+\boldsymbol{b}_{3}\right)$ $\boldsymbol{k}_{14}{ }^{(3)}=-\square\left(\boldsymbol{b}_{1}+\boldsymbol{b}_{2}-\boldsymbol{b}_{3}\right)$

$\left\{\begin{array}{c}\boldsymbol{k}_{15}{ }^{(1)}=\square\left(\boldsymbol{b}_{1}+\boldsymbol{b}_{2}\right)+\square \\ \boldsymbol{b}_{3} \\ \boldsymbol{k}_{15}^{(2)}=-\boldsymbol{k}_{15}(1)\end{array}\right.$ $\boldsymbol{k}_{4}^{(1)}=\square \boldsymbol{b}_{3}$

$\boldsymbol{k}_{4}{ }^{(2)}=\square \boldsymbol{b}_{2}$ $\boldsymbol{k}_{4}^{(3)}=\square \boldsymbol{b}_{1}$

Tetragonal $\mathrm{P}\left(\Gamma_{q}\right)$

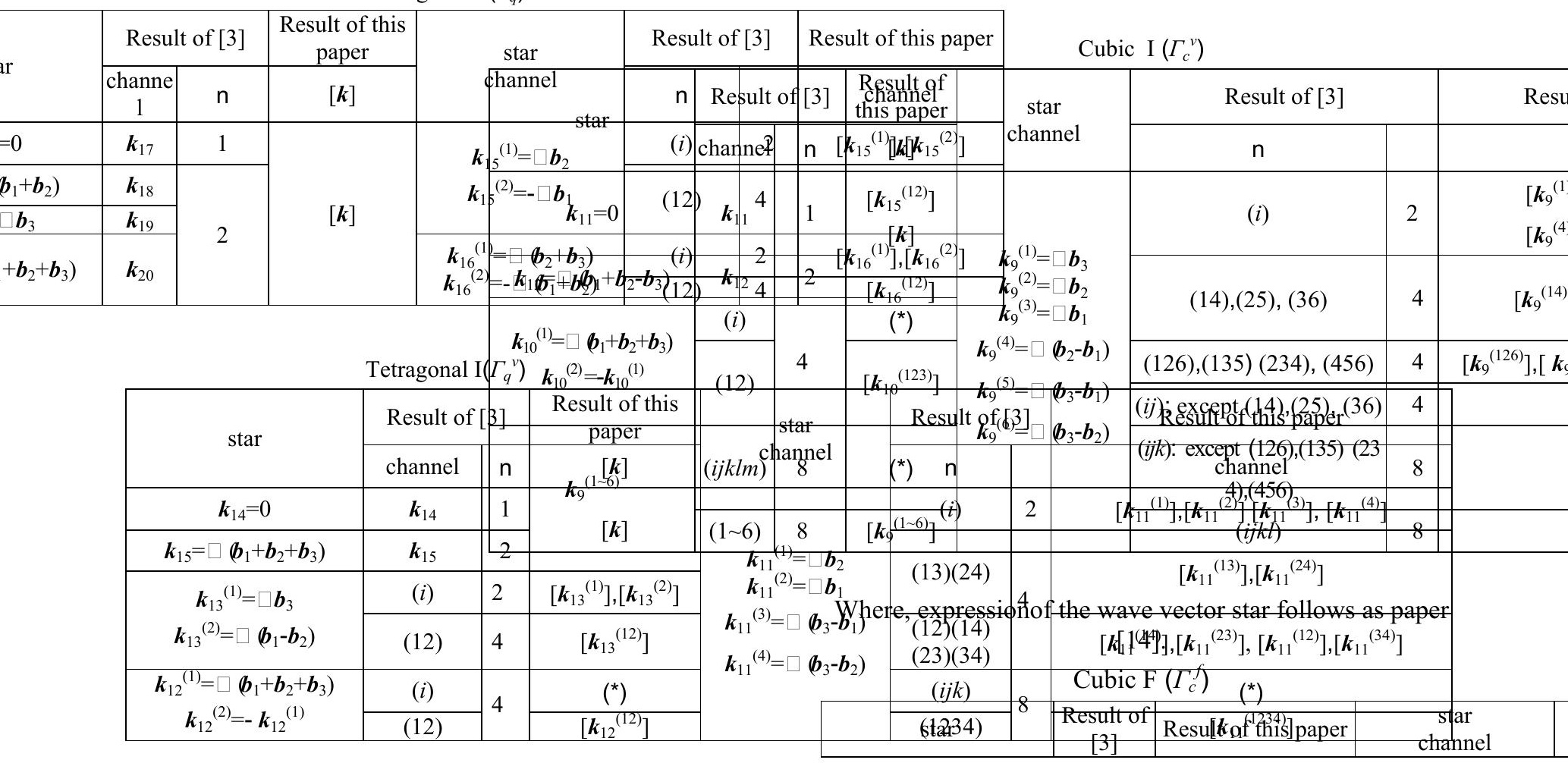

\section{Volume 5 Issue 7, July 2016 www.ijsr.net}

Licensed Under Creative Commons Attribution CC BY 


\section{International Journal of Science and Research (IJSR) \\ ISSN (Online): 2319-7064}

Index Copernicus Value (2013): 6.14 | Impact Factor (2015): 6.391

\begin{tabular}{|c|c|c|c|c|}
\hline & $\begin{array}{c}\text { channe } \\
1\end{array}$ & $n$ & {$[k]$} & \\
\hline $\boldsymbol{k}_{11}=0$ & $\boldsymbol{k}_{11}$ & 1 & {$[k]$} & \multirow{9}{*}{$\begin{array}{c}\boldsymbol{k}_{8}{ }^{(1)}=\square\left(\boldsymbol{b}_{1}+\boldsymbol{b}_{2}\right. \\
\square\left(\boldsymbol{b}_{2}+\boldsymbol{b}_{3}\right) \\
\boldsymbol{k}_{8}^{(2)}=-\boldsymbol{k}_{8}{ }^{(1)} \\
\boldsymbol{k}_{8}^{(3)}=\square\left(\boldsymbol{b}_{1}+\boldsymbol{b}_{3}\right. \\
\square\left(\boldsymbol{b}_{1}+\boldsymbol{b}_{2}\right) \\
\boldsymbol{k}_{8}^{(4)}=-\boldsymbol{k}_{8}^{(3)} \\
\boldsymbol{k}_{8}^{(5)}=\square\left(\boldsymbol{b}_{2}+\boldsymbol{b}_{3}\right. \\
\square\left(\boldsymbol{b}_{1}+\boldsymbol{b}_{3}\right) \\
\boldsymbol{k}_{8}^{(6)}=-\boldsymbol{k}_{8}{ }^{(5)}\end{array}$} \\
\hline \multirow{3}{*}{$\begin{array}{l}\boldsymbol{k}_{10}^{(1)}=\square\left(\boldsymbol{b}_{1}+\boldsymbol{b}_{2}\right) \\
\boldsymbol{k}_{10}{ }^{(2)}=\square\left(\boldsymbol{b}_{1}+\boldsymbol{b}_{3}\right) \\
\boldsymbol{k}_{10}{ }^{(3)}=\square\left(\boldsymbol{b}_{2}+\boldsymbol{b}_{3}\right)\end{array}$} & (i) & 2 & $\begin{array}{l}{\left[\boldsymbol{k}_{10}{ }^{(1)}\right]} \\
{\left[\boldsymbol{k}_{10}{ }^{(2)}\right]} \\
{\left[\boldsymbol{k}_{10}{ }^{(3)}\right]}\end{array}$ & \\
\hline & (ij) & 4 & $\left({ }^{*}\right)$ & \\
\hline & (123) & & {$\left[\boldsymbol{k}_{10}{ }^{(123)}\right]$} & \\
\hline \multirow{5}{*}{$\begin{array}{c}\boldsymbol{k}_{9}^{(1)}=\square\left(\boldsymbol{b}_{1}+\boldsymbol{b}_{2}+\boldsymbol{b}_{3}\right) \\
\boldsymbol{k}_{9}^{(2)}=\square \boldsymbol{b}_{1} \\
\boldsymbol{k}_{9}^{(3)}=\square \boldsymbol{b}_{2} \\
\boldsymbol{k}_{9}^{(4)}=\square \boldsymbol{b}_{3}\end{array}$} & (i) & 2 & $\begin{array}{l}{\left[\boldsymbol{k}_{9}{ }^{(1)}\right],\left[\boldsymbol{k}_{9}{ }^{(2)}\right]} \\
{\left[\boldsymbol{k}_{9}{ }^{(3)}\right],\left[\boldsymbol{k}_{9}{ }^{(4)}\right]}\end{array}$ & \\
\hline & (ij) & 4 & $\begin{array}{l}{\left[\boldsymbol{k}_{9}^{(12)}\right],\left[\boldsymbol{k}_{9}{ }^{(13)}\right]} \\
{\left[\boldsymbol{k}_{9}^{(14)}\right],\left[\boldsymbol{k}_{9}{ }^{(23)}\right]} \\
{\left[\boldsymbol{k}_{9}^{(24)}\right],\left[\boldsymbol{k}_{9}{ }^{(34)}\right]}\end{array}$ & \\
\hline & $(i j k)$ & & $\left({ }^{*}\right)$ & \\
\hline & $(1 \sim 4)$ & 8 & {$\left[\boldsymbol{k}_{9}^{(1234)}\right]$} & \\
\hline & & & & \\
\hline
\end{tabular}

In the fourth, eighth column of table-2, symbol (*) denotes the star channel not to be tolerated during the spontaneous symmetry breaking. $n$ is the volume change.Also, for example, $\left[\boldsymbol{k}_{10}{ }^{(1)}\right]$ denotes the star channel related to first arm of Lifshitz star $\boldsymbol{k}_{10},\left[\boldsymbol{k}_{10}{ }^{(12)}\right]$ expresses the star channel related to both first, second arms of Lifshitz star $\boldsymbol{k}_{10}$.

As shown, the star channels tolerated in previous papers are not tolerable according to our result. This means that not arbitrary combination of the arms of the given star, but only selected combination of the arms which is satisfied symmetry of parent phase is possible during the translational symmetry breaking ${ }^{[12,15,16]}$.

\section{Wave vector star channel Group}

If the symmetry breaking is related to the wave vector star channel $[\boldsymbol{k}]_{i}$, we can write the change $\delta \rho$ in the distribution density function as the formula (2).

$$
\delta \rho_{[k]_{i}}=\sum_{k, l} \eta_{l}^{k} \varphi_{l}^{k}
$$

Where $\varphi_{l}^{\boldsymbol{k}}$ is the basis function related to arms of star taking part in the phase transition among the basis functions of the irreducible representation of the space group of the formula (2). Thus, $\varphi_{l}^{\boldsymbol{k}}$ are not to concern the full irreducible representation any more. The change $\delta \rho$ in the distribution density function in the formula (2) and (5) is both characterized the lower symmetry phases. But in the formula (2), $\delta \rho(\boldsymbol{r})$ is associated with all the arms of star.
On the other hand, in the formula (5), chammegl is associated with the arms of star taking part in the ${ }_{\star}$ phase transition actually. Thus, the properties of the phase transition are essentially different. (12),(34),(56)

From this, the lower symmetry phas $\left[\boldsymbol{k}_{8}\left({ }_{6}^{(56)}\right]_{4,4}\right.$ related to the

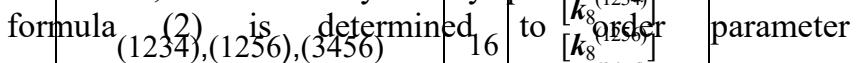
$\boldsymbol{\eta}_{\alpha}=\left(\eta_{1}, \eta_{2}, \ldots, \eta_{m}\right)$ corresponding to th $\boldsymbol{k}_{8}{ }^{(34) 94]}$ irreducible

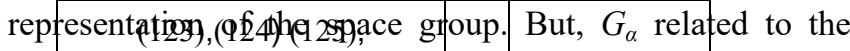
ormula (5126), desermiges), to order parameter $\boldsymbol{\eta}_{\beta}=\left(\eta_{1}, \eta_{2}, \ldots\right.$,

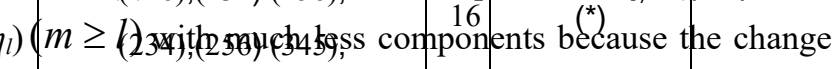
of the depsitis), (flabctipz) is only decomposed by the basis function related to arms of the star channel. In other words, if we consider),(thy (syinmmetry breaking in the view of the waye vector star $_{2}$ hannels, we will have the order parameter $\left(\boldsymbol{b}_{2}+\boldsymbol{b}_{3}\right) \eta_{\beta}$ but not the order parameter $\eta_{k .} 16$ (25),(26) (35), If element\$36),(45f) (Hre) space group act on $\delta q_{[k]_{i}}$ of the

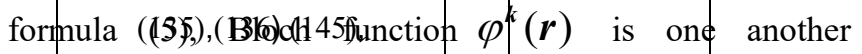

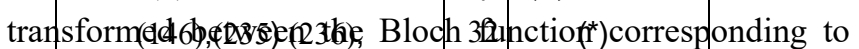
the arms of the $s^{2 t a r}$ channel. Then, the channel $[\boldsymbol{k}]=\{\boldsymbol{k}\}$ having all the arms of star is one another transformed when

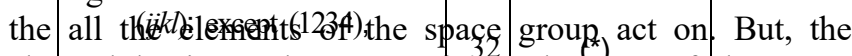
channel havings6)(3456) some of the elements of the space group is one angther transformed when the some of the elements of the space group act on

$$
\text { (123456) }
$$

$$
32 \quad\left[\boldsymbol{k}_{8}^{(123456)}\right]
$$

As is known, the set of elements of space group $G_{0}$ leaving one arm of wave vector star invariant is called wave vector group or little group. Likewise, we can newly define the set of elements of space group $G_{0}$ leaving the wave vector star channel invariant. We will call it the channel group of wave vector star or simply channel group and expressed to $G_{[k]}$.

$$
G_{[\boldsymbol{k}]}=\left\{g_{n} \mid g_{n}[\boldsymbol{k}]=[\boldsymbol{k}]+\boldsymbol{b}\right\}
$$

or

$$
G_{\left[\cdots, \boldsymbol{k}_{i}, \cdots, \boldsymbol{k}_{j}, \cdots\right]}=\left\{g_{n} \mid g_{n}\left[\cdots, \boldsymbol{k}_{i}, \cdots, \boldsymbol{k}_{j}, \cdots\right]=\left[\cdots, \boldsymbol{k}_{i}+\right.\right.
$$

The star channel group can be seen as the group in which the wave vector group is generalized to the channel. So between the space group related to all the arms of star, the star channel group and the little group, a group-subgroup relationship exists.

The star channel group can be written as follows when we know the little group $G_{\boldsymbol{k} \mathrm{i}}$ related to each arms of the star.

$$
G_{[\boldsymbol{k}]}=G_{\left[\boldsymbol{k}_{1}, \boldsymbol{k}_{2}, \cdots, \boldsymbol{k}_{\mathrm{n}}\right]}=\left\{G_{\boldsymbol{k}_{1}} \cap G_{\boldsymbol{k}_{2}} \cap \cdots \cap G_{\boldsymbol{k}_{\mathrm{n}}}\right\} \cup\left\{g_{l}\right\}(8)
$$

First member $\left\{G_{\boldsymbol{k}_{1}} \cap G_{\boldsymbol{k}_{2}} \cap \cdots \cap G_{\boldsymbol{k}_{\mathrm{n}}}\right\}$ of the formula (8) is corresponded to a unit element and second member $\left\{g_{l}\right\}$ is corresponded to the elements of the permutation group except a unit element. That is,

$$
\left(\begin{array}{llll}
\boldsymbol{k}_{1}, & \boldsymbol{k}_{2}, & \cdots, & \boldsymbol{k}_{\mathrm{n}} \\
\boldsymbol{k}_{1}, & \boldsymbol{k}_{2}, & \cdots, & \boldsymbol{k}_{\mathrm{n}}
\end{array}\right)=(e)=\left\{G_{\boldsymbol{k}_{1}} \cap G_{\boldsymbol{k}_{2}} \cap \cdots \cap G_{\boldsymbol{k}_{\mathrm{n}}}\right\}
$$

\section{Volume 5 Issue 7, July 2016 www.ijsr.net}




\section{International Journal of Science and Research (IJSR) \\ ISSN (Online): 2319-7064}

Index Copernicus Value (2013): 6.14 | Impact Factor (2015): 6.391

$$
\begin{gathered}
\left(\begin{array}{cccc}
\boldsymbol{k}_{1}, & \boldsymbol{k}_{2}, & \cdots, & \boldsymbol{k}_{\mathrm{n}} \\
\boldsymbol{k}_{i}, & \boldsymbol{k}_{j}, & \cdots, & \boldsymbol{k}_{l}
\end{array}\right)=(i, j, \cdots, l)=\left\{\begin{array}{c}
g_{l} \boldsymbol{k}_{1}=\boldsymbol{k}_{i} \\
g_{l} \boldsymbol{k}_{2}=\boldsymbol{k}_{j} \\
\vdots \\
g_{l} \boldsymbol{k}_{\mathrm{n}}=\boldsymbol{k}_{l}
\end{array}=\left\{g_{l}\right\}\right. \\
(i, j, \ldots, l=\overline{1})
\end{gathered}
$$

In table-3, we showed the possible wave vector star channel groups of $\operatorname{star} \boldsymbol{k}_{8}$ in crystals belonging to a face-centered cubic lattice. In third, fourth column of table -3 , the symbol of the rotation elements follows to paper $\left[{ }^{19}\right]$. Wave vector star $\boldsymbol{k}_{8}$ consists of six arms.

Table 3: Channel point groupsofstar $\boldsymbol{k}_{8}$ of face-centered cubic lattice

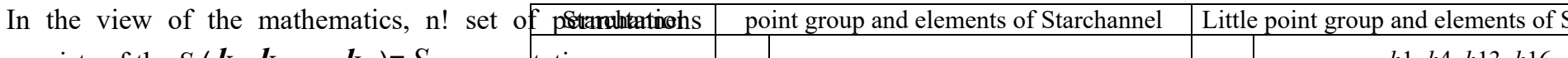
consists of the $S_{n}\left(\boldsymbol{k}_{1}, \boldsymbol{k}_{2}, \ldots, \boldsymbol{k}_{n}\right) \equiv S_{\boldsymbol{k}_{n}}$ permutation grepp.

$$
\left(\begin{array}{llll}
\boldsymbol{k}_{1}, & \boldsymbol{k}_{21}, & \cdots, & \boldsymbol{k}_{\mathrm{n}} \\
P_{\boldsymbol{k}_{1},} & P_{\boldsymbol{k}_{2}}, & \cdots, & P_{\boldsymbol{k}_{\mathrm{n}}}
\end{array}\right)=\left(\begin{array}{l}
\boldsymbol{k}_{(34)} \\
{\left[\boldsymbol{k}_{8}(345)\right](9)} \\
P_{\boldsymbol{k}_{(33}}
\end{array}\right.
$$

In formula (7), the elements of the space ${ }^{2}$ group

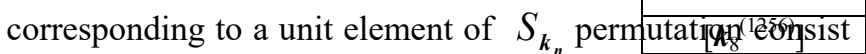
of the group. This group is expressed as follows.

$$
G_{k_{1}} \cap G_{k_{2}} \cap \cdots \cap \cap G_{k_{\mathrm{n}}}=G_{e}(10)
$$

Then, $G_{e}$ is obviously invariant subgroup.

Because the group $G_{e}$ is invariant subgroup, the channel group is decomposed as follows.

$$
G_{[k]}=g_{0} G_{e}+g_{1} G_{e}+\ldots+g_{\mathrm{n}} G_{e}(11)
$$

\begin{tabular}{c|c|c}
\hline \multirow{2}{*}{$\begin{array}{c}h 1 \sim h 4, h 13 \sim h 16, \\
h 25 \sim h 28, h 37 \sim h 40\end{array}$} & $\boldsymbol{D}_{2 d}$ & $h 1, h 4, h 13, h 16$, \\
& & $h 26, h 27, h 38, h 39$ \\
\cline { 2 - 3 } & $\boldsymbol{C}_{2 v}$ & $h 1, h 3, h 26, h 28$ \\
\hline \multirow{3}{*}{$h 1 \sim h 4, h 21 \sim h 24}$, & $\boldsymbol{D}_{2 d}$ & $h 1, h 3, h 21, h 23$, \\
$h 25 \sim h 28, h 45 \sim h 48$ & & $h 26, h 28, h 46, h 48$ \\
\cline { 2 - 3 } & $\boldsymbol{C}_{2 v}$ & $h 1, h 4, h 26, h 27$ \\
\hline \multirow{2}{*}{$h 1 \sim h 4, h 17 \sim h 20}$, & $\boldsymbol{D}_{2 d}$ & $h 1, h 2, h 17, h 18$, \\
$h 25 \sim h 28, h 41 \sim h 44$ & & $h 27, h 28, h 43, h 44$ \\
\cline { 2 - 3 } & $\boldsymbol{C}_{2 v}$ & $h 1, h 4, h 26, h 27$ \\
\hline \multirow{2}{*}{$h 1 \sim h 48$} & $\boldsymbol{D}_{2 d}$ & $h 1, h 4, h 13, h 16$, \\
& & $h 26, h 27, h 38, h 39$ \\
\hline
\end{tabular}

Thus, we can define the factor groupwhich the channel group $G_{[k]}$ divided by $G_{e}$. That is,

$$
G_{f}=G_{[k]} / G_{e}(12)
$$

Factor group $G_{f}=G_{[k]} / G_{e}$ is generally isomorphic the subgroup of $n$-demonsional permutation group $S_{k_{n}}$.

Since the star channel group is also a space group, we can also define the point group and the transition group of the star channel.

Star channels group $G_{[k]}$ has transration group $T_{[k]}$ which is an invariant subgroup.We call this group $T_{[k]}$ the translation group of the star channel.

$G_{[k]}$ is decomposed to left cocets of $T_{[k]}$ as follows.

$$
G_{[k]}=g_{1} T_{[k]}+g_{2} T_{[k]}+\cdots+g_{s} T_{[k]}
$$

Because $T_{[k]}$ is an invariant subgroup of $G_{[k]}$, we can define the factor group $G_{[k]} / T_{[k]}$. We call this the point group of the star channel $G_{[k]}^{0}$.

Star channel group $G_{[k]}$ can be used to consider directly the symmetry of the phase transition during various spontaneous symmetry breaking.

Suppose that the phase transition $G_{0} \leftrightarrow G_{1} \leftrightarrow G_{2} \ldots \leftrightarrow G_{\mathrm{n}}$ occurs in crystal.If $G_{n}$ is related to some of arms of the given star channel, we can consider that lower symmetry phases $G_{1}, G_{2}$, $\ldots, G_{n}$ are obtained from the star channel group $G_{[k] n}$ during the phase transition $G_{[k] n} \leftrightarrow G_{1} \leftrightarrow G_{2} \leftrightarrow \ldots \leftrightarrow G_{n}$.
As shown, the star channels taking part in the translational symmetry breaking have two, four and six arms channel of star. On the other hand, the point group of two arms channel $\left[\boldsymbol{k}_{8}^{(12)}\right]$ of star, four arms channel[ $\left.\boldsymbol{k}_{8}{ }^{(3456)}\right]$ of star is both $D_{4 h}$. Thus, we can know that the number of arms in the star channel is different but their star channel groups are isomorphic. Also, we can know that there is a group-subgroup relationship between the channel group of all the arms and some of the arms of star.

To conclude, if two arms of given star take part in the phase transition, we can consider the star channel group of the tetragonal lattice associated with two arms which is simpler than the space of the cubic lattice associated with all the arms. Thus, we can sure that the star channel group can be used more effectively in studying the phase transition ${ }^{[17,18]}$.

\section{Bravais Lattice Types}

In studying the superstructure phases, it is also important to correctly know the lattice types determined by it besides the information about the arms of wave vector star.

For exactly finding out the lattice types of the superstructure phases, we must know three problems.

First, we must exactly know the crystal system of the lattice.In this paper, we found out it by means of the star channel point group and its possible subgroups.

To select the crystal system means that the point group of the possible lower symmetry phase is determined in the view of the group theory. Meanwhile, each Bravais lattices must be invariant to translation group because the crystal has the translational symmetry. The translation group is related to various star channels of the wave vector star and the point group of latticeis isomorphic the channel point 


\section{International Journal of Science and Research (IJSR) \\ ISSN (Online): 2319-7064}

Index Copernicus Value (2013): 6.14 | Impact Factor (2015): 6.391

group $G_{[k]}^{f}=G_{[k]}^{0}=G_{[k]} / T_{[k]}$. After all, the point groups of the possible lower symmetry during the spontaneous symmetry breaking of the crystal are isomorphic to the subgroups of the point group $G_{[k]}^{0}$ of the star channel associated with a given wave vector star.So, we found out the crystal system of the possible Bravias lattice during the translational symmetry breaking byttifireafigintof thelejpants of channel point group are its subgrouph.

Second, we must exactly know the vector orienthation $\Gamma^{v}\left\{\begin{array}{l}\{1,13 \sim 16 \\ \text {, }\end{array}\right.$ Bravais lattice.

We found out it by means of the translation g®oup of the channel and its kernel of the representation.

The crystal lattice of the new phase is characterized by translation vectors $t_{\mathrm{D}}$ satisfying the equatiom $, 16,25$ $\exp \left(i \boldsymbol{k}_{\alpha} \boldsymbol{t}_{\mathrm{D}}\right)=1$ with respect to the given wave $\left.2 h v e c t o r, 28,37,40\right\}$ channel $\left[\boldsymbol{k}_{\alpha}\right]$.The set of these translation vectors belong to representation kernelker $\Gamma(\boldsymbol{t})$ which-are invariant subgroups of the translation group $T_{[k]}$ of the star channel.Among thes, 25,26$\}$ translation vectors $\boldsymbol{t}_{\mathrm{D}}$, the vectors $\boldsymbol{d}_{[k]}=\left\{d_{1}, d_{2}, d_{3}\right\}$ having th, $\left.1,25,27\right\}$ shortest length become the vectors of Bravais unit of the lower symmetry phase.

The volumn change of a unit cell during the phase translation is expressed $n=T_{[\boldsymbol{k}]} / \operatorname{ker} \Gamma_{(, t}^{h}, C_{2 h} \quad\{1,13,25,37\}$ having the space of a face-centered cubic lattice as parent symmetry phase and showed the some of them in the picture 1.

Table 4: Phase transition channel of star channel $\left[\boldsymbol{k}_{8}{ }^{(1}\right.$ ${ }^{2)}$ ] of space group $O_{h}^{5}$, cubic $\mathrm{F}$

Number of

phase transition channel

$\mathrm{P}_{16}{ }^{1}-(1,2)$ $-\left(a_{1}, a_{2}, 2 a_{3}\right), 2-\left(a_{2}\right.$, - $-\left(a_{1}, a_{2}, 2 a_{3}\right), 2-\left(a_{2}, a\right.$ 3- $\left(2 a_{3}, a_{2}, a_{1}\right), 4-\left(2 a_{3}\right.$ 5- $\left(a_{1}, 2 a_{3}, a_{2}\right), 6-\left(a_{2}\right.$ $P_{16}^{2}-(1 \sim 6)$ $\{1,4,25,28\}$

Third, we must find out the possible Bravais latiee types during the phase translation.

We found out the various subgroups of the star channel point group and obtained the Brayais lattice types $\boldsymbol{d}_{[k] \mathrm{s}}\left\{1, \mathrm{that}^{25,40\}}\right.$ were invariant to them.We just define the Bravais lattice types that are invariant to the subgroups $G_{[k] s}^{0}$ of the star channel point group as lattice channel $\Gamma_{\text {and }}$ exfress like $\left.d_{[k] \mathrm{s}} 25\right\}$ $(\mathrm{s}=0,1, \ldots \mathrm{s})$

Generally one wave vector star channel has several lattice channels.So we can define the phase transition channel as the sum of the star channel and its lattice channels phase transition channel=star channel+lattice channel. Conveniently, the phase transition channel is expressed to $P_{[k]}^{d_{[k] s}}$.Here $i$ is the number of the wave vector star channel $[\boldsymbol{k}], s$ is the number of lattice channel $\boldsymbol{d}_{[k] \mathrm{s}}$ of the given star channel $[\boldsymbol{k}], j$ is the number of various orientations of the translation vectors ina lattice channel.

The phase transition $P_{[k]}^{d_{[k] s}}$ means that the lower symmetry phase has the $j$ th lattice types of the $s$ th lattice channel when the phase transition occurs along the $i$ th star channel in crystal.

In this paper, we also obtained the phase transition channels, that is, 179 wave vector star channels and lattice channels associated with it.

For example, in table-4, we showed the phase transition channels associated with the star channel $\left[\boldsymbol{k}_{8}{ }^{(12)}\right]$ in crystal 


\section{International Journal of Science and Research (IJSR) \\ ISSN (Online): 2319-7064}

Index Copernicus Value (2013): 6.14 | Impact Factor (2015): 6.391

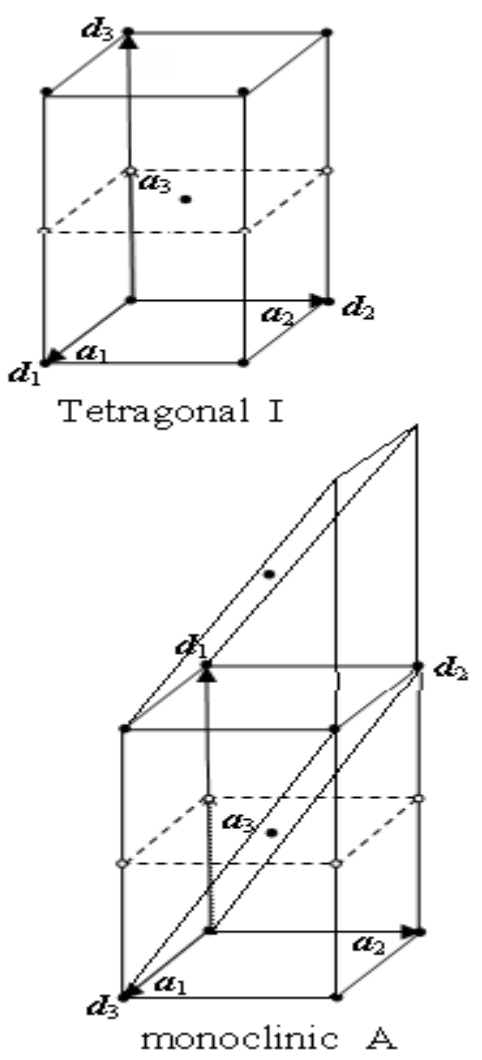

Picture- 1some oflattice channels of star channel $\left[\boldsymbol{k}_{8}^{(12)}\right]$ of space group $O_{h}^{5}$, cubic $\mathrm{F}$

With respect to various subgroups of the point group of the star channel $\left[\boldsymbol{k}_{8}{ }^{(12)}\right]$, five lattice types exist, that is, a body-centered tetragonal lattice, a body-centered orthorhombic lattice, a face-centered orthorhombic lattice, monoclinic lattice, triclinic lattice. In the phase transition channels, we showed particularly the elements of point group of the possible Bravais lattice and its vector orientations.

In this paper, we defined the star channel and lattice types unlike in the previous paper. Then, we can use efficiently it in studying the tranlational symmetry breaking. The wave vector star channel has been widely used in obtaining the distribution function of the atoms and the theoretical diffraction intensity in the superstructure. Also, by means of the lattice channel, we can know the crystallographic properties of such as the lattice types and the orientation of the translation vectors associated with the given star channel.

\section{Conclusion}

In this paper, we have suggested a kind of the new method for determining the wave vector star channel in close relation with symmetry of order parameter and investigated the mutual relationship between all the possible wave vector star channel and Bravais lattice types. Also, we have for the first time defined the conception of the channel group of the wave vector star, which can be efficiently used in studying the translational symmetry breaking related to all the Lifshiz wave vector star of 230 space groups. .

First, we established a method to obtain the wave vector star channel by accepting the new order parameter space spanned by representation of translation group and by finding the subspaces invariant to the image of the representation.

Second, channel group of the wave vector star was defined for the for the first time and it was shown that the possible Bravais lattice types can be obtained by it.

Third, it was shown that the conception of the phase transition channel as the whole-embracing concept of wave vector star channel and lattice channel can be used efficiently in studying the phase transition of spontaneous symmetry breaking types.

Fourth, we also obtained the wave vector star channel, the star channel group and the lattice channels associated with all the Lifshiz stars of 230 space groups.

\section{References}

[1] E.M.Lifshitz, Zh. Eksp. Teor. Fiz.,11,255(1941)

[2] V.E. Naish et al., Kristallografiya,21,1085(1976)

[3] Yu. A. Izyumov, Phase Transition and Crystal Symmetry, Kluwer, 1990

[4] Yu. A. Izyumov, V.E. Naish, R. P. Ozerov., Neutronographiya Magneticov., Moscow, 312, 1981

[5] O.V.Gurin et al., Jour. Less. Domm. Metals, 101, 529(1984)

[6] A. I. Gusev and A. A. Rempel, Structural Phase Transition in Non-Stoichiometric Compouns, Izd. Nauka,308,1988(in Russian)

[7] A.I.Gusev et al.,Phys. Stat.Sol(a),135(1),15(1993)

[8] A. A. Rempel, Us. Fiz. Nauk,166,1,33-62(1996)

[9] M. I. Solobieba et al., Izv. Byis. Uzbek. Zav. Fiz., 4,43(2002)

[10]D. A. Dabyidob et al., Fiz. Tverd. Tela., 51, 1, 147 154(2009)

[11] M. V. Jaric et al., J. Math. Phys. 24, 12(1983)

[12] Il Hwan Kim et al., Bull. Acad. Sci. D.P.R.K., 2, 27(2001)

[13]Il Hwan Kim et al., Bull. Acad. Sci. D.P.R.K., 4, 25(1995)

[14] C. J. Bradley et al., The Mathrmatical Theory of symmetry in Solids, (Clarendon Oxford), 1972

[15] W. Cao et al., Phys. Rev., B64, 024106(2001)

[16] D.M.Hatch et al., Phys. Rev., B65, 094110(2002)

[17] Il Hwan Kim et al., Bull. Acad. Sci. D.P.R.K., 5, 32(2006)

[18] Il Hwan Kim et al., Physica B, 424, 20-26(2013)

[19] O. V. Kovalev, Representation of the Crystallographic Space Group, Irreducible Representation, Induced Representations and Co-representation, 365, 1993 